\title{
Medical infrared imaging and orthostatic analysis to determine lameness in the pelvic limbs of dogs
}

\author{
Erika Fernanda V. Garcia ${ }^{1}$, Catherine A. Loughin ${ }^{1, *}$, Dominic J. Marino ${ }^{1}$, Joseph Sackman ${ }^{1}$, Scott E. Umbaugh ${ }^{2}$, \\ Jiyuan $\mathrm{Fu}^{2}$, Samrut Subedi ${ }^{2}$, Martin L. Lesser ${ }^{3}$, Meredith Akerman ${ }^{3}$ and João Eduardo W. Schossler ${ }^{4}$ \\ ${ }^{1}$ Department of Surgery, Long Island Veterinary Specialists, 163 South Service Road, Plainview, NY 11803, USA \\ ${ }^{2}$ Computer Vision and Image Processing Laboratory, Electrical and Computer Engineering Department, Southern \\ Illinois University at Edwardsville, Edwardsville, IL 62026-1801, USA \\ ${ }^{3}$ North Shore - LIJ Health System Feinstein Institute for Medical Research, Biostatistics Unit, Manhasset, NY 11030, \\ USA \\ ${ }^{4}$ Universidade Federal de Santa Maria, Santa Maria, Brazil
}

\begin{abstract}
Subtle lameness makes it difficult to ascertain which is the affected limb. A study was conducted to investigate a change in the thermal pattern and temperature of the thermal image of the paw print in a lame pelvic limb compared to a non-lame pelvic limb of dogs confirmed by orthostatic analysis. Fourteen client owned dogs with a unilateral pelvic limb lameness and 14 healthy employee dogs were examined and the pelvic limbs radiographed. Thermal images of the paw print were taken after each dog was kept in a static position on a foam mat for 30 seconds. Average temperatures and thermographic patterns were analyzed. Analysis was performed in a static position. The asymmetry index for each stance variable and optimal cutoff point for the peak vertical force and thermal image temperatures were calculated. Image pattern analysis revealed $88 \%$ success in differentiating the lame group, and $100 \%$ in identifying the same thermal pattern in the healthy group. The mean of the peak vertical force revealed a $10.0 \%$ difference between the left and right pelvic limb in healthy dogs and a $72.4 \%$ between the lame and non-lame limb in the lame dog group. Asymmetry index analysis revealed 5\% in the healthy group and 36.2\% in the lame group. The optimal cutoff point for the peak vertical force to determine lameness was $41.77 \%$ (AUC $=0.93$ ) and for MII $0.943 \%$ $(A U C=0.72)$. The results of this study highlight the change in the thermal pattern of the paw print in the lame pelvic limb compared to a non-lame pelvic limb in the lame group and the healthy group. Medical infrared imaging of the paw prints can be utilized to screen for the lame limb in dogs.
\end{abstract}

Keywords: Cruciate, Infrared imaging, Lameness, Orthostatic analysis.

\section{Introduction}

Examination of the gait is the first diagnostic step in the evaluation of a patient with a lameness (Griffon, 2008), and relies on the assessment of asymmetry (Budsberg et al., 1993; Fanchon and Grandjean, 2007). Watching a dog walk is subjective, and it can be difficult to differentiate left versus right sided lameness if asymmetry is subtle (Fanchon and Grandjean, 2007; Voss et al., 2007).

Force plate analysis has been used for a wide range of kinetic applications in veterinary medicine, and its use has been proven as a valuable tool in gait analysis (Anderson, 1994; Voss et al., 2007; Gillette and Angle, 2008). This analysis provides a noninvasive, objective and quantitative evaluation of the forces occurring between the paw and the ground during the stance phase of the stride (Anderson, 1994), as well as the pressure distributions of the paw in a standing position (orthostatic pattern) (Barbosa et al., 2011). However, the complicated logistics, high cost of the equipment, and laborious interpretation makes this method typically inaccessible in most clinics for small animals. Medical infrared imaging (MII) has proven to be a sensitive method for detecting changes in superficial temperature of the skin (Turner, 2001). This noncontact, non-invasive diagnostic technique measures heat emitted from a body surface as infrared radiation (Van hoogmoed and Snyder, 2002). The infrared image is a color map of warm colors representing areas of elevated temperature (e.g. associated with inflammation, increased circulation or metabolic rate), and cool colors representing areas of decreased tissue temperature or perfusion (e.g. vascular shunt, infarction or change in autonomic nervous system) (Eddy et al., 2001; Turner, 2001; Van hoogmoed and Snyder, 2002). The change in superficial heat detected by the infrared camera is related to sympathetic nerve control of skin blood flow and the increase and decrease of postganglionic pressure that regulates microdermal blood flow. 
Medical infrared imaging has been reported mostly for use in large animals, such as llamas, cattle, and horses to aid in the diagnosis of infectious (Colak et al., 2008; Rainwater-Lovett et al., 2009), reproductive (Purohit et al., 1985), orthopedic (Purohit and McCoy, 1980; Eddy et al., 2001), and neurologic conditions (Purohit et al., 1980; Purohit, 2008). Recently, studies in small animals have been published evaluating normal thermal patterns in limbs of healthy dogs (Loughin and Marino, 2007), differentiation of cranial cruciate ligament deficient stifles from normal in dogs (Infernuso et al., 2010), assessing for intervertebral disc disease in chondrodystrophic dogs (Grossbard et al., 2014), assessing for elbow dysplasia in dogs (McGowan et al., 2015), and to assist in detection of hyperthyroidism in cats (Waddell et al., 2015). Another study in cats (Vainionpaa et al., 2013) revealed that MII of the paw prints can detect a change in weight bearing. In this study, the author observed a change in the paw print thermal image, suggesting a painful process in the limb of the cat. Palpation of the cat and a questionnaire filled out by the owner were used for comparison to the images, but no other diagnostics were performed to support the suspicion of a pain or diagnose a specific disease process.

The purpose of this study was to investigate a change in the thermal pattern and temperature of the thermal image of the paw print in a lame pelvic limb compared to a non-lame pelvic limb in dogs with cranial cruciate ligament rupture. We also evaluated the thermal images of the paw prints of non-lame pelvic limbs of healthy dogs for comparison purposes, and used orthostatic analysis to objectify the data. We hypothesized that MII could be used to detect changes in the thermal pattern and temperatures of the thermal image of paw prints in dogs with a documented lameness as compared to the non-lame limb and the limbs of healthy dogs.

\section{Criteria for case selection}

\section{Materials and Methods}

Client-owned dogs (>18 kg) that presented between January through July 2013 for unilateral pelvic limb lameness due to cranial cruciate ligament rupture (CCLR) were included in this study. The diagnosis of unilateral CCLR was determined by clinical history, orthopedic examination, and radiographic evaluation (radiographs of the pelvis, bilateral stifles and hocks were reviewed) and was confirmed by exploratory surgery of the stifle.

Thermal imaging was performed before induction of anesthesia for surgery. A board-certified surgeon assessed the lameness and classified it with a clinical lameness grade (Table 1) (Witte and Scott, 2011). Written consent from the owner was acquired for each dog included in the study. Dogs were excluded if another orthopedic or neurologic condition was diagnosed.
Table 1. Lameness grade at a walk and trot.

\begin{tabular}{|c|c|}
\hline Grade & Description \\
\hline 0 (None) & No lameness is observed at a walk or trot \\
\hline 1 (Mild) & $\begin{array}{l}\text { Lameness is present, but may only be } \\
\text { consistently apparent at a trot }\end{array}$ \\
\hline $\begin{array}{l}2 \text { (Mild to } \\
\text { moderate) }\end{array}$ & $\begin{array}{l}\text { Mild lameness is obviously present at a } \\
\text { walk and is worse at a trot }\end{array}$ \\
\hline 3 (Moderate) & Obvious lameness is present at both gaits \\
\hline $\begin{array}{l}4 \text { (Moderate } \\
\text { to severe) }\end{array}$ & $\begin{array}{l}\text { Obvious lameness is present at both gaits } \\
\text { and may be intermittently non-weight } \\
\text { bearing }\end{array}$ \\
\hline 5 (Severe) & $\begin{array}{l}\text { Lameness is non-weight bearing most or } \\
\text { all of the time }\end{array}$ \\
\hline
\end{tabular}

\section{Control group}

Healthy employee-owned dogs with absence of orthopedic and neurologic disease assessed via physical examination and radiographic evaluation of the hips, stifles and tarsus were selected. Written consent from the owner was acquired for each control dog in the study. Dogs were excluded from the study if an orthopedic or neurologic condition was diagnosed.

\section{Infrared Imaging}

All the thermal images were obtained in the same room with an ambient temperature of $21^{\circ} \mathrm{C}$ that is controlled by a centralized climate control system. The dogs were housed in runs at the same temperature. All thermal images were obtained before sedation or general anesthesia. A portable infrared camera (Med 2000 IRIS, Meditherm Inc., Fort Meyers, FL) with a silicone microbolometer with a spectral range between 7 and 14 $\mu \mathrm{m}$, resolution $160 \times 120$ pixels with a focal plane array amorphous, and an emissivity setting of one $(\mathrm{e}=1)$ was used. The camera was connected to a laptop computer for real-time data analysis. The dogs were positioned symmetrically as possible on a foam mat in a static standing position. The dog was kept in this position for 30 seconds, while the handler remained beside the dog to facilitate positioning. If the dog moved the limbs before 30 seconds, the dog was again positioned. After the 30 seconds, the dog was allowed to walk forward, and the thermographer captured images of the paw prints left by the pelvic limbs on the foam mat. To capture these images, the camera was held approximately $1.5 \mathrm{~m}$ directly over the mat.

Each image was saved within the software program (WINTES 2, Compix Inc., Lake Oswego, OR) for further evaluation and review. The program was preset for a temperature range of $8^{\circ} \mathrm{C}$ with a 16 -shade color map. The color map indicated warmer temperatures as white and red and cooler temperatures as blue and black. Each image was focused and saved by the operator of the computer. After each image was saved, 
the temperature scale was adjusted so that the color range of the image was balanced. The program saved the final image with the specific $8^{\circ} \mathrm{C}$ temperature range for each image as a tiff file.

\section{Force plate orthostatic analysis}

Orthostatic analysis was performed in the same room as MII. Two permanently mounted force plates (AMTImodel\# OR6-7-1000, 18.25x20x3.25 in, Advanced Mechanical Technology Inc, Watertown, MA) placed lengthwise on a platform and connected to a computer equipped with a software program (Acquire version $7.33 \mathrm{~V}$, Advanced Mechanical Technology Inc, Watertown, MA) designed for data collection and storage were used.

The force plates were embedded in a carpeted runway 1.16 meters long and 0.45 meters wide. The dog was placed in a standing, static position as symmetrically and naturally as possible so that each pelvic limb was on one of the force plates. The dog remained in this position for 5 seconds for data collection. A minimum of three valid trials for each dog was used to obtain mean peak vertical force (PVF) values. Peak vertical forces were expressed in percent of body weight $(\% \mathrm{BW})$. The asymmetry index (ASI) for each gait variable for each dog was calculated by use of the following equation (Fanchon and Grandjean, 2007):

(|XR - XL|/ [|XR + XL|x 0.5]) x 100

Where $\mathrm{XR}$ is the mean of a given gait variable for static peak force $(100 * \mathrm{~N} / \mathrm{N})$ of the right pelvic limb and the $\mathrm{XL}$ is the mean of a given gait variable for static peak force $(100 * \mathrm{~N} / \mathrm{N})$ of the left pelvic limb. According to this method, an ASI of $0 \%$ indicates perfect gait symmetry for the measured variable, whereas positive or negative values indicate right or left hind limb asymmetry, respectively (Voss et al., 2007).

\section{Imaging Pattern Analysis}

An image processing software program (CVIPtools, Computer Vision and Image Processing Laboratory, Department of Electrical and Computer Engineering, School of Engineering, Southern Illinois University, Edwardsville, IL) that included a collection of computer vision and image processing routines was used to analyze images.

Ten sets of experiments were performed with the thermal images. The left and right side were not separated. Four color normalization methods were used along with the original RGB images. Three data normalization were used on the feature data, soft-max, standard normal density as well as the original raw feature data.

The experiments were performed with the Computer Vision and Image Processing - Feature extraction and Pattern Classification (CVIP-FEPC), with each set having 2047 permutations, and using Nearest Neighbor (NN) and Train/Test Set as the classification methods; histogram, spectral and texture features with a pixel distance of six. The testing method used was the leaveone-out method.

\section{Statistical analysis}

For each of the analyses, standard logistic regression (PROC LOGISTIC, SAS Institute Inc., Cary, NC) was used, where the predictor value was either absolute percent difference in peak force (using orthostatic analysis) or absolute percent difference in thermal images, and the outcome variable was whether or not the dog was lame.

Absolute percent difference was calculated as follows: $\%$ difference $=(($ absolute value $(($ Right - Left $) /($ Right + Left)/2)))*100)/2

An optimal rule was defined as the rule corresponding to the point on the receiver operating characteristic (ROC) curve closest to $(0,1)$ (i.e. closest to $100 \%$ sensitivity and $0 \%$ false positive rate). The area under the ROC curve (AUC) was calculated separately for each predictor variable. The ROC comparisons were performed using a contrast matrix to take differences of the areas under the empirical ROC curves (DeLong et al., 1988).

A result was considered statistical significant at the $\mathrm{p}<0.05$ level of significance. All analyses were performed using SAS version 9.3. The mean peak vertical force and asymmetry indexes were also calculated to compare the healthy and lame groups.

\section{Results}

Fourteen lame dogs ( 9 female spayed, 1 female intact and 4 males neutered) with confirmed CCLR were included in the study. Nine dogs had a lameness associated with the left pelvic limb and five dogs in the right pelvic limb. Breeds represented were 5 mixedbreed dogs, 3 Pit Bulls, 2 Labrador Retrievers; and 1 each Golden Retriever, Boxer, Portuguese Water Dog and Shetland Sheepdog. The mean age was 4.7 years old (range 1 to 9 years, median 4.7 years) and mean body weight was $29.5 \mathrm{~kg}$ (range 19.9 to $43.5 \mathrm{~kg}$, median $28.9 \mathrm{~kg}$ ). Six dogs had grade 4 lameness, two dogs had grade 3 , three dogs had grade 2 , and three dogs had grade 1 . The normal dogs consisted of fourteen dogs (8 female spayed and 6 male neutered), 6 were mixed breed dogs, 2 Boxers, and 1 each Pit Bull, Doberman, Australian Cattle Dog, Greyhound, German Shepherd Dog and Labradoodle. The mean age was 4.9 years old (range 0.8 to 9.7 years, median 4 years) and mean body weight was $28.3 \mathrm{~kg}$ (range 18.8 to $41.7 \mathrm{~kg}$, median 26.9 $\mathrm{kg}$ ). The paw print image temperatures were separated into the following groups: healthy dogs (right limb versus left limb), lame dogs (lame limb versus nonlame limb), lame limb (lame dog) versus normal limbs (healthy dogs) and non-lame limb (lame dog) versus normal limb (healthy dogs). In the lame dog group, the temperature difference between the paw print thermal image of the lame limb versus the non-lame limb revealed a difference in temperature of $0.5^{\circ} \mathrm{C}$ (Table 2). 
Table 2. Average, $\pm \mathrm{SD}$, minimum and maximum of temperatures $\left({ }^{\circ} \mathrm{C}\right)$ of the paw print obtained in healthy (left and right pelvic limb) and lame dogs (lame limb and nonlame pelvic limb).

\begin{tabular}{llccc} 
Dog & Limb & Mean \pm SD & $\begin{array}{c}\text { Avg } \\
\min \end{array}$ & $\begin{array}{c}\text { Avg } \\
\max \end{array}$ \\
\hline Healthy & Left limb & $21.67 \pm 1.3$ & 20.5 & 22.8 \\
Dogs & Right limb & $21.82 \pm 1.3$ & 20.7 & 23.1 \\
Lame & Lame limb & $21.08 \pm 1.2$ & 20.5 & 21.7 \\
Dogs & Non-lame limb & $21.61 \pm 1.2$ & 20.6 & 23.0 \\
\hline
\end{tabular}

In the healthy dog group, the temperature difference between the right and left limb was $0.1{ }^{\circ} \mathrm{C}$. Image pattern analysis revealed an $88 \%$ success rate in differentiating the paw print thermal image in the lame pelvic limb versus the non-lame limb (lame group). For the paw print thermal image of the right pelvic limb versus the left pelvic limb (healthy group) the success rate was 100\%. Fig. 1 (lame group) and Fig. 2 (healthy group) show these image pattern differences.

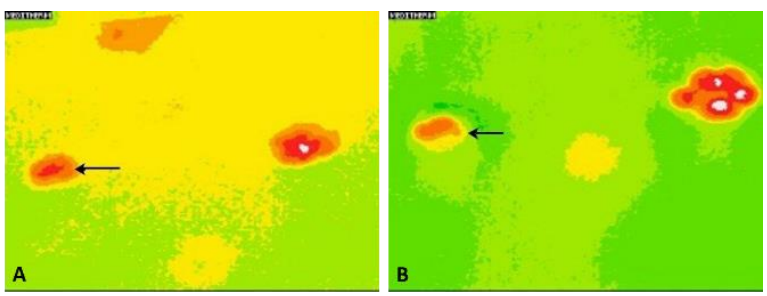

Fig. 1. (A): Thermal image of the paw print in a dog with grade 2 lameness (black arrow). (B): Thermal image of the paw print in a dog with grade 4 lameness (black arrow). Note the difference in the temperature in the paw print of the lame limb between figures $1 \mathrm{~A}$ and $1 \mathrm{~B}$.

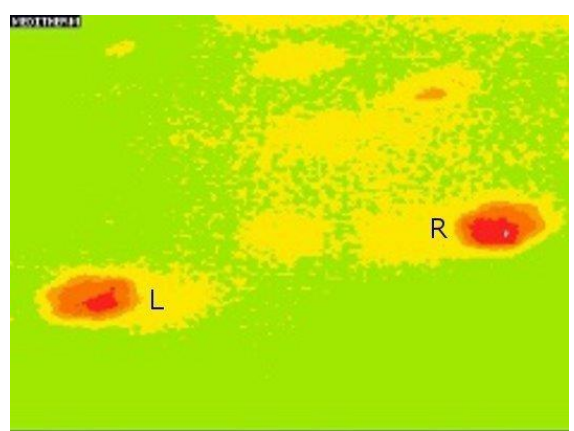

Fig. 2. Thermal image of the paw prints in a healthy $\operatorname{dog}(\mathrm{L}=$ left, $\mathrm{R}=$ right side).

The mean of the peak vertical force $(100 * \mathrm{~N} / \mathrm{N})$ showed a $10.0 \%$ difference between left $(20.9 \pm 2.1)$ and right $(18.1 \pm 2.9)$ limb in the healthy dogs. In the lame dogs, the difference between the lame limb $(5.4 \pm 6.4)$ and the non-lame limb $(30.0 \pm 5.0)$ was $72.4 \%$. Asymmetric index analysis showed that there are significant differences between the healthy group $(5 \%)$ and the lame group (36.2\%) ratings (Table 3 ).
Table 3. Ground reaction forces (mean \pm SD), average (avg) of the percentage $(\%)$ difference of the peak vertical force (PVF) in static position of the healthy and lame dogs and asymmetry index (ASI) of the healthy and lame dogs.

\begin{tabular}{lcccc}
\cline { 2 - 4 } & \multicolumn{2}{c}{ Healthy Dogs mean } & \multicolumn{2}{c}{ Lame Dogs mean } \\
\cline { 2 - 4 } & Left limb & Right limb & $\begin{array}{c}\text { Lame } \\
\text { limb }\end{array}$ & $\begin{array}{c}\text { Non-lame } \\
\text { limb }\end{array}$ \\
\hline $\begin{array}{l}\text { PVF } \\
(100 * \mathrm{~N} / \mathrm{N})\end{array}$ & $20.94 \pm 2.1$ & $18.05 \pm 2.9$ & $5.44 \pm 6.4$ & $30.01 \pm 5.0$ \\
$\begin{array}{l}\text { Avg \% } \\
\text { difference* }\end{array}$ & $10.0 \%$ & $72.4 \%$ \\
ASI $(\%)$ & $5 \%$ & $36.2 \%$ \\
\hline
\end{tabular}

*(Avg \% difference): The average of all trials for each patient was calculated and then the percentage difference between the left and right was calculated.

Based on the ROC curve using percent difference in peak force (Fig. 3), the optimal cutoff point was determined to be $41.8 \%$ with an $\mathrm{AUC}=0.93$. Using this cutoff point for percent difference in peak force, the sensitivity $=78.6 \%$ and the specificity $=100.0 \%$.

Based on the ROC curve using percent difference in thermal imaging (Fig. 4), the optimal cutoff point was determined to be $0.943 \%$ with an $\mathrm{AUC}=0.72$. Using this cutoff point for percent difference in peak force, the sensitivity $=64.3 \%$ and the specificity $=78.6 \%$.

The AUC for orthostatic analysis was significantly greater than for thermal imaging (difference $=0.21$, $\mathrm{p}<0.032$ ). Figure 5 shows the ROC curves for comparison.

\section{Discussion}

The present study evaluated the use of MII to detect lameness in dogs by the change in the thermal pattern and lower temperature of the paw prints. Image pattern analysis was successful in differentiating the paw print thermal image of the lame limb versus the non-lame limb in lame dogs.

The only other comparative study (Vainionpaa et al., 2013), revealed an uneven thermal pattern in the images of cat paw prints associated with differences in weight bearing, but did not use any objective data analysis to support their findings. In this study, lameness was detected by the temperature difference observed in the paw print thermal image of the dog, and confirmed with force plate orthostatic analysis.

This information in conjunction with a physical examination and gait assessment may be able to assist with confirmation of a lame limb in situations where the lameness is subtle or subclinical, but further studies with larger groups of dogs with a subtle or subclinical lameness would need to be done to confirm this. Further diagnostics such as anatomical MII, radiographs, computed tomography or magnetic resonance imaging could then be performed to assess for a more specific diagnosis. 


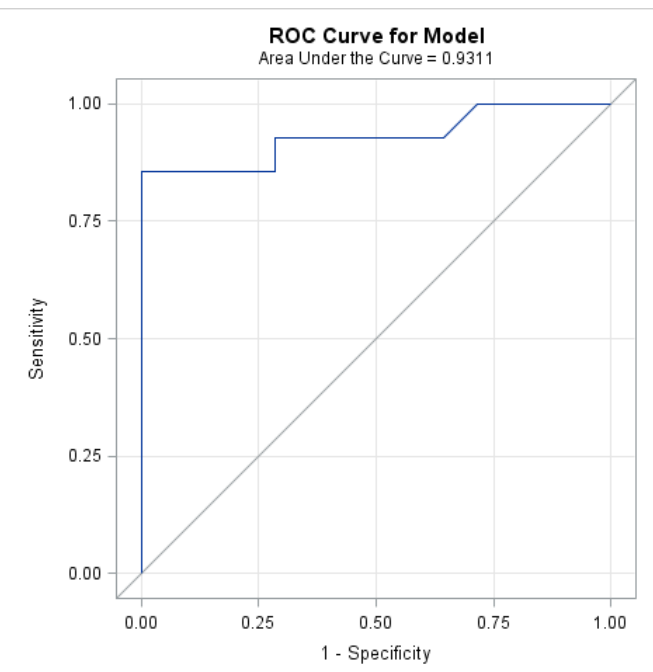

Fig. 3. ROC curve for percent difference in peak force.

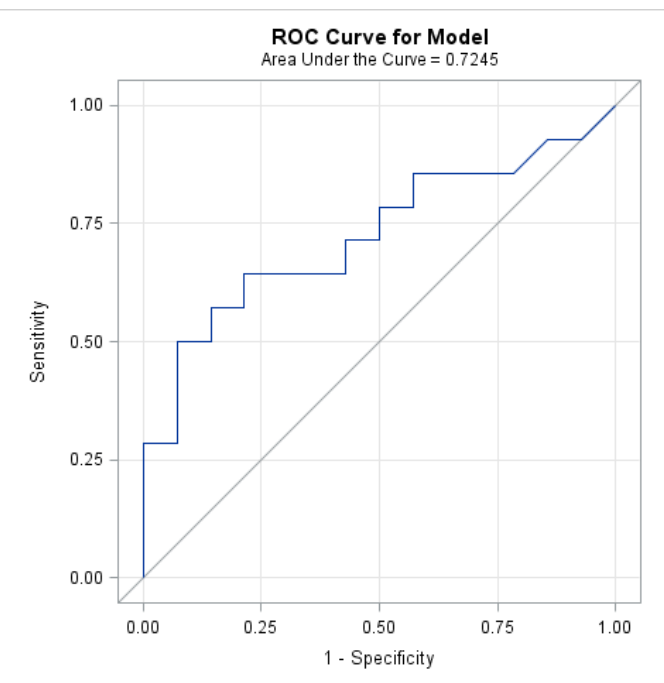

Fig. 4. ROC curve for percent difference in MII.

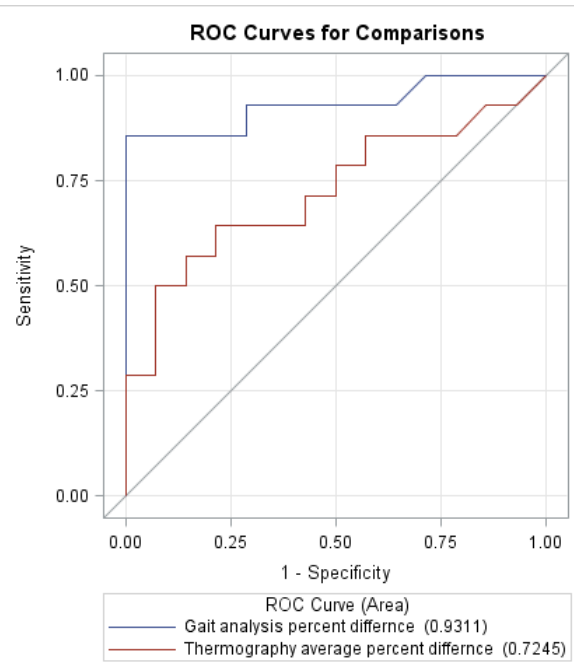

Fig. 5. ROC curves for comparison.
Temperature analysis revealed an increase of $0.5^{\circ} \mathrm{C}$ between the paw prints of the lame dogs lame limb versus the non-lame limb, and in the healthy dogs the difference was $0.1^{\circ} \mathrm{C}$ between the right and left limbs. Studies in healthy humans, horses, and dogs have shown symmetry between right and left sides with temperature differences a fracture of a degree centigrade (Uematsu et al., 1988; Loughin and Marino, 2007; Purohit, 2008; Westermann et al., 2013). Studies of clinical disease have shown that a difference greater than $1^{\circ} \mathrm{C}$ of temperature between two of the same anatomic regions indicate an abnormality such as inflammation (Purohit et al., 1985; Turner, 1991, 2001; Eddy et al., 2001). The comparison of this data with the thermal paw print image would not be possible because it comes from different surfaces. However, when reviewing the data from the imaging pattern analysis and the orthostatic analysis between healthy and lame dogs, the data supports differences in weight bearing in lame versus non-lame limbs in lame dogs and similarities in data between the non-lame limbs. In this case, we argue that the difference of $0.5^{\circ} \mathrm{C}$ in the temperature of the paw print between the pelvic limbs is actually significant.

When evaluating the data for the lame pelvic limb in dogs the value of the temperature difference is less than the temperature in healthy dogs, the significance becomes stronger when we observe the thermal pattern differences, where the color distribution is remarkably different. The ability to differentiate between lame and healthy dogs using force plate orthostatic analysis increases with the severity of the lameness (Voss et al., 2007), which is supported by the AUC of 0.93 for the percent difference in the peak force. The optimal cutoff for the peak force was abnormally high due to 2 dogs with a higher percent difference in peak force. This could be explained by the difficulty in positioning some of the dogs on the force plates and keeping them in place for a measurement. Dogs move and many of them will lean toward the handler. Kinetic gait analysis studies have analyzed variation due to handlers, acclimation and day-to-day variations (Rumph et al., 1997, 1999), which supports this falsely elevated number.

Similar observations were made with the thermal images where it was noted that the higher the grade of lameness, the greater the color change (cooler color pattern), and the paw print became smaller. The AUC of 0.72 was not as high as for peak force, but is still considered supportive of MII of the paw prints as a viable option to help determine lameness. In the study with cats, irregular heat patterns were observed in paw prints by the change in weight bearing of cats that showed signs of discomfort during palpation; however, they showed no significant lameness (Vainionpaa et al., 2013). Taking both studies into consideration, it is 
possible that MII could be useful in screening cases of subtle lameness to determine the affected limb, but since we only had 3 dogs with a subtle lameness in this study further investigation would be necessary.

In this study, the temperature of the paw print was observed on a foam mat. The thermal pattern was detected from the transfer of heat by conduction (Eddy et al., 2001; Turner, 2001) from the paw to the foam mat, and the camera detected the print by radiation emission from the mat.

Emissivity of the object is the ability to absorb and emit infrared radiation (Eddy et al., 2001; Turner, 2001). This is an important concept in thermal imaging, because the ability of a material to reflect or emit heat must be considered in the interpretation of an image. The thermal camera generates images based on the amount of heat generated rather than reflected; meaning, they actually detect differences in temperatures of the target and surroundings (Eddy et al., 2001). The foam mat was chosen because the thermal conductivity was high, besides being inexpensive and easily accessible.

There was no significant asymmetry in the paw print observed by temperature and imaging pattern analysis in healthy dogs. All species studied thus far have provided remarkable bilateral symmetrical patterns of infrared emission (Purohit and McCoy, 1980; Loughin and Marino, 2007; Purohit, 2008). The high degree of right-to-left symmetry is a valuable asset in the diagnosis of a unilateral problem associated with various inflammatory disorders (Loughin and Marino, 2007; Purohit, 2008). In gait analysis, the assessment of the asymmetry index is a good method to evaluate lameness, because it is based on right versus left comparisons with modification of the balance of the dog (Fanchon and Grandjean, 2007). In this study, the orthostatic analysis revealed $5 \%$ asymmetry in the healthy dogs, which is not significant. These results are consistent with another study (Budsberg et al., 1993), in which the author found in the vertical symmetry indices a deviation of $<8 \%$ from perfect for all variables tested, reflecting near perfect symmetry in the vertical axis.

The clinical lameness score is used routinely in veterinary medicine as part of the orthopedic examination. Mild lameness cannot always be detected when the dog walks but may become evident at a trot (Witte and Scott, 2011). In the present study, data from the lameness score was helpful and, although not the objective of this study, it was possible to compare the degree of lameness with the changes in size and temperature of the thermal image of the paw print of the dogs. Future studies with a larger number of animals may produce more accurate results.

The limitation of the present study was the ability to keep the dog in a standing position for 30 seconds, especially the dogs with lameness. The time was arbitrarily determined, but during the course of the study it was observed that it might be possible to obtain images in less time. Another limitation was the low number of dogs, decreasing the statistical power. This was most likely due to the strict selection criteria of no orthopedic or neurologic deficits in the pelvic limbs. Many clinical patients have contralateral cruciate tears or hip dysplasia, which made them ineligible for this study. It may be beneficial to conduct additional studies with different weights, breeds of dogs, and contralateral disease to assess the changes in thermal patterns.

Based on the results obtained, we concluded that there was a change in the thermal pattern of the paw print in the lame pelvic limb compared to non-lame pelvic limb in lame and limbs of healthy dogs. Medical infrared imaging could be useful as a screening method, allowing the targeting of a disease or injury to a specific limb or region of interest. Further investigations are required to specify variations of the paw print in different orthopedic conditions.

\section{Acknowledgments}

This paper was supported in part by CNPq, Conselho Nacional de Desenvolvimento Científico e Tecnológico - Brazil. The authors thank Dr. Peter Leando for technical assistance.

\section{Conflict of interest}

The authors declare that there is no conflict of interest.

\section{References}

Anderson, M.A. 1994. Force plate analysis: a noninvasive tool for gait evaluation. Comp. Cont. Ed. Pract. Vet. 16, 857-867.

Barbosa, A.L.T., Schossler, J.E.W., Bolli, C.M., Lemos, L.F.C. and Medeiros, C. 2011. Test and standardize of force plate in orthostatic pattern in dogs. Arq. Bras. Med. Vet. Zootec. 63, 559-566.

Budsberg, S.C., Jevens, D.J., Brown, J., Foutz, T.L., DeCamp, C.E. and Reece, L. 1993. Evaluation of limb symmetry indices, using ground reaction forces in healthy dogs. Am. J. Vet. Res. 54, 15691574.

Colak, A., Polat, B., Okumus, Z., Kaya, M., Yanmaz, L.E. and Hayirli, A. 2008. Short communication: early detection of mastitis using infrared thermography in dairy cows. J Dairy Sci. 91:42444248.

DeLong, E.R., DeLong, D.M. and Clarke-Pearson, D.L. 1988. Comparing the areas under two or more correlated receiver operating characteristic curves: a nonparametric approach. Biometrics 44, 837-845.

Eddy, A.L., Van hoogmoed, L.M. and Snyder, J.R. 2001. The role of thermography in the management of equine lameness. Vet. J. 162, 172-181.

Fanchon, L. and Grandjean, D. 2007. Accuracy of asymmetry indices of ground reaction forces for 
diagnosis of hind limb lameness in dogs. Am. J. Vet. Res. 68, 1089-1094.

Gillette, R.L. and Angle, T.C. 2008. Recent developments in canine locomotor analysis: a review. Vet. J. 178, 165-176.

Griffon, D.J. 2008. Canine gait analysis: a decade of computer assisted technology. Vet. J. 178, 159-160.

Grossbard, B.P., Loughin, C.A., Marino, D.J., Marino, L.J., Sackman, J., Umbaugh, S.E., Solt, P.S., Afruz, J., Leando, P., Lesser, M.L. and Akerman, M. 2014. Medical infrared imaging (thermography) of type I thoracolumbar disk disease in chondrodystrophic dogs. Vet. Surg. 43, 869-876.

Infernuso, T., Loughin, C.A., Marino, D.J., Umbaugh, S.E. and Solt, P.S. 2010. Thermal imaging of normal and cranial cruciate ligament-deficient stifles in dogs. Vet. Surg. 39, 410-417.

Loughin, C.A. and Marino, D.J. 2007. Evaluation of thermographic imaging of the limbs of healthy dogs. Am. J. Vet. Res. 68, 1064-1069.

McGowan, L., Loughin, C.A., Marino, D.J., Umbaugh, S.E., Liu, P., Amini, M., Solt, P., Lesser, M.L. and Akerman, M. 2015. Medical Infrared Imaging of Normal and Dysplastic Elbows in Dogs. Vet. Surg. 44, 874-882.

Purohit, R.C. 2008. Use of thermography in veterinary medicine. In: Lee HMN CJ, ed. Rehabilitation Medicine and Thermography. Wilsonville, OR: Impress Publications, pp: 129-144.

Purohit, R.C., Hudson, R.S., Riddell, M.G., Carson, R.L., Wolfe, D.F. and Walker, D.F. 1985. Thermography of the bovine scrotum. Am. J. Vet. Res. 46, 2388-2392.

Purohit, R.C. and McCoy, M.D. 1980. Thermography in the diagnosis of inflammatory processes in the horse. Am. J. Vet. Res. 41, 1167-1174.

Purohit, R.C., McCoy, M.D. and Bergfeld, W.A. 3rd. 1980. Thermographic diagnosis of Horner's syndrome in the horse. Am. J. Vet. Res. 41, 11801182.

Rainwater-Lovett, K., Pacheco, J.M., Packer, C. and Rodriguez, L.L. 2009. Detection of foot-and-mouth disease virus infected cattle using infrared thermography. Vet. J. 180, 317-324.
Rumph, P.F., Steiss, J.E. and Montgomery, R.D. 1997. Effects of selection and habituation on vertical ground reaction force in greyhounds. Am. J. Vet. Res. 58, 1206-1208.

Rumph, P.F., Steiss, J.E. and West, M.S. 1999. Interday variation in vertical ground reaction force in clinically normal Greyhounds at the trot. Am. J. Vet. Res. 60, 679-683.

Turner, T.A. 1991. Thermography as an aid to the clinical lameness evaluation. Vet. Clin. North Am. Equine Pract. 7, 311-338.

Turner, T.A. 2001. Diagnostic thermography. Vet. Clin. North Am. Equine Pract. 17, 95-113.

Uematsu, S., Edwin, D.H., Jankel, W.R., Kozikowski, J. and Trattner, M. 1988. Quantification of thermal asymmetry. Part 1: Normal values and reproducibility. J. Neurosurg. 69, 552-555.

Vainionpaa, M.H., Raekallio, M.R., Junnila, J.J., Hielm-Björkman, A.K., Snellman, M.P. and Vainio, O.M. 2013. A comparison of thermographic imaging, physical examination and modified questionnaire as an instrument to assess painful conditions in cats. J. Feline Med. Surg. 15, 124-131.

Van hoogmoed, L.M. and Snyder, J.R. 2002. Use of infrared thermography to detect injections and palmar digital neurectomy in horses. Vet. J. 164, 129-141.

Voss, K., Imhof, J., Kaestner, S. and Montavon, P.M. 2007. Force plate gait analysis at the walk and trot in dogs with low-grade hindlimb lameness. Vet. Comp. Orthop. Traumatol. 20, 299-304.

Waddell, R.E., Marino, D.J., Loughin, C.A., Tumulty, J.W., Dewey, C.W. and Sackman, J. 2015. Medical infrared thermal imaging of cats with hyperthyroidism. Am. J. Vet. Res. 76, 53-59.

Westermann, S., Buchner, H.H., Schramel, J.P., Tichy, A. and Stanek, C. 2013. Effects of infrared camera angle and distance on measurement and reproducibility of thermographically determined temperatures of the distolateral aspects of the forelimbs in horses. J. Am. Vet. Med. Assoc. 242, 388-395.

Witte, P. and Scott, H. 2011. Investigation of lameness in dogs: 2. hindlimb. In Practice 33, 58-66. 